\title{
Pathogenesis and management of diabetic foot ulcers
}

\author{
Yousif Alsanawi $^{1}{ }^{*}$, Hassan Alismail $^{1}$, Mustafa AlabdRabalnabi ${ }^{1}$, Hattan Alturki ${ }^{2}$, \\ Abdullah Alsuhaibani ${ }^{3}$, Mamdouh Mahbub ${ }^{4}$, Abdullah Junayd ${ }^{5}$, Abdulraheem Alamri ${ }^{6}$, \\ Mujahid Jawahraji $^{4}$, Mohammed Aldaghmani ${ }^{7}$
}

\author{
${ }^{1}$ Imam Abdulrahman Bin Faisal University, Dammam, Saudi Arabia \\ ${ }^{2}$ Poznan University of Medical Sciences, Poznan, Poland \\ ${ }^{3}$ King Saud bin Abdulaziz University for Health Sciences, Riyadh, Saudi Arabia \\ ${ }^{4}$ Taif University, Taif, Saudi Arabia \\ ${ }^{5}$ Umm AlQura University, Mecca, Saudi Arabia \\ ${ }^{6}$ King Khaled University, Abha, Saudi Arabia \\ ${ }^{7}$ Aljouf University, Aljouf, Saudi Arabia
}

Received: 25 September 2018

Revised: 11 October 2018

Accepted: 12 October 2018

\section{*Correspondence:}

Dr. Yousif Alsanawi,

E-mail: usersef55@hotmail.com

Copyright: (c) the author(s), publisher and licensee Medip Academy. This is an open-access article distributed under the terms of the Creative Commons Attribution Non-Commercial License, which permits unrestricted non-commercial use, distribution, and reproduction in any medium, provided the original work is properly cited.

\begin{abstract}
Diabetes mellitus is a major healthcare issue worldwide, and the trends keep rising. Diabetic foot ulcer is a morbidity associated with the disease process and causes significant impairment in quality of life due to its severe complications including infection, gangrene, and amputation. In this study, we aim to understand the pathogenesis of diabetic foot ulcer, its complications, and management strategies. We conducted this review using a comprehensive search of MEDLINE, PubMed and EMBASE from January 1970 to March 2017. The following search terms were used: diabetic foot ulcer, complications of diabetes mellitus, foot gangrene, surgical debridement of foot ulcer, osteomyelitis. Diabetic foot ulcer is a common morbidity in patients with diabetes mellitus, which can lead to lower limb amputation unless a prompt, rational, multidisciplinary approach to therapy is taken. Proper management can ensure successful and fast healing which includes patient education, blood glucose control, wound debridement, advanced dressing, offloading, and surgery.
\end{abstract}

Keywords: Diabetic foot ulcer, Ulcer debridement, Diabetes care

\section{INTRODUCTION}

Diabetes mellitus (DM) is considered a major issue in healthcare system worldwide. Data shows that in the past 2 decades there has been increasing trends in the incidence of DM. According to recent studies, the number of patients diagnosed with DM has increased from 30 million cases in 1985 to 285 million in 2010. It is expected that more that 360 million people by $2030 .^{1}$

Diabetic patients have a higher risk of different morbidities like diabetic foot ulcer (DFU). DFU is one of the most common morbidities of DM that has shown an increasing rate in the last decade. Overall, it is expected that more than $15 \%$ of diabetics will develop DFU. Even though accurate data are difficult to estimate the prevalence of DFU, the prevalence of DFU is expected to be between $4 \%-27 \%$. $^{2}$ The rates of hospitalization due to DFU has increased, it has been considered a leading cause of hospitalization in diabetic patients. Approximately $20 \%$ of hospital admissions among diabetics are due to DFU. DFU can present with a wide range of complications such as infection, gangrene, amputation, and even death without treatment. 
Amputations are not uncommon morbidities in DFU. The rates of lower limb amputations in diabetics is estimated to be 15 times higher than the general population. ${ }^{3}$

\section{METHODS}

\section{Data sources and search terms}

We conducted this review using a comprehensive search of MEDLINE, PubMed and EMBASE, from January 1970 to March 2017. The following search terms were used: diabetic foot ulcer, complications of diabetes mellitus, foot gangrene, surgical debridement of foot ulcer, osteomyelitis.

\section{Data extraction}

Two reviewers have independently reviewed the studies, abstracted data and disagreements were resolved by consensus. Studies were evaluated for quality and a review protocol was followed throughout.

\section{Pathogenesis}

It is believed that DFU has multiple etiologies; the main etiologies are peripheral neuropathy ischemia from peripheral vascular disease.

\section{Neuropathy}

Neuropathy affects the nervous system in diabetics in many manifestations including motor, autonomic, and sensory. Affection of the motor fibers of the intrinsic foot muscles can lead to an inequity between flexion and extension of the affected foot. As a result, it leads to foot deformities which stimulates the osteocytes of the bones to leading to bony prominences and pressure points, that gradually break the dermatological layers of the skin causing ulcerations and infections. ${ }^{4}$

Autonomic nerves control sweating. Autonomic neuropathy affects the process of sweating. As a result, the skin becomes dry and prone to fissures which allow the bacteria to invade the deep layers of the dermis leading to infection, abscesses and even sepsis. This is exacerbated by the loss of sensory nerve fibers. When trauma occurs, the nervous system is not able to detect the insult. Therefore, trauma goes unnoticed and the wounds progress and the injury spreads due to repetitive pressure and shear forces from weight bearing and ambulation. ${ }^{4}$

Peripheral neuropathy can also cause Charcot arthropathy. Charcot arthropathy occurs because of muscle and joint laxity that leads to changes in the alignment of feet ligaments leading to changes in the arches of the feet. The mechanism is not different than DFU. It is the outcome of a of motor, autonomic, and sensory neuropathies. Moreover, ischemia of arterial supply of the smooth muscle leads to bone demineralization which can exacerbate the arthropathy. ${ }^{5}$

\section{Peripheral vascular disease}

Atherosclerosis in diabetics is not different than the general population, it starts as fatty depositions that progress to plaques. Affections of the coronary arteries, cerebral vessels, and peripheral vessels are the major manifestations of macrovascular disease in DM. ${ }^{6}$

Peripheral vascular disease (PVD) affects all the arteries, however atheroma tends to occur in specific anatomical sites, especially at the bifurcations of the vessels, where shear forces are much less. Bifurcations in the lower limb are the aortoiliac part and the superficial femoral artery (SFA) in the adductor canal. In patients with diabetes the distal vessels are more prone to injury below the biifurcation including the peroneal, anterior, and posterior tibialis. However, the dorsalis pedis artery is not affected in most cases. ${ }^{6}$

\section{Foot infection}

Patients with DM are more prone to infections due to lower immunity, hyperglycemia, and ischemia. Infections of the diabetic foot can have major consequences because deep infections are more common because of the anatomical deformities.

The anatomy of the foot consists of connected compartments. As a result, Infection of one compartment can lead to the spread of the bacteria to other compartments causing infections in other sites. The lack of pain sensation due to neuropathy allows the patient to ambulate which facilitates the spreading of the infections. Infection of the soft tissues such as the plantar aponeurosis, tendons, muscle sheaths and fascia can lead to a higher rate of spreading. ${ }^{7}$

\section{Osteomyelitis}

Osteomyelitis in patients with DM is often challenging. It occurs when the infection from a contiguous site to the deep tissues of the foot reaching the cortex to the bone marrow. The diagnosis of osteomyelitis is often difficult in diabetic patients, because it is hard to differentiate between soft tissue infection, bone infection and noninfectious etiologies such as charcot foot. ${ }^{8}$

Different imaging studies are used to guide the diagnosis of osteomyelitis such as X-rays; often shows focal osteopenia, cortical erosions or periosteal reaction in the early stage and sequestration in the late stage. Probing is a simple clinical test used to aid the diagnosis at office; A sterile metal probe is inserted into the ulcer if it penetrates to the bone it usually confirms the diagnosis of osteomyelitis. To confirm the diagnosis; a bone biopsy with culture and sensitivity is often sensitive and specific. 
Newer imaging studies are sometimes useful such as nuclear bone scan, computerized tomography scan (CT), positron emission tomography (PET), and magnetic resonance imaging (MRI). ${ }^{9}$

\section{MANGMENT OF DFU}

\section{Feet examination}

The physical examination of the feet is essential in all diabetic patients, it should be conducted on every visit. Physicians should carefully look for any changes in skin such as dryness, fissures, callus, deformities varicose veins and nail changes. Interdigital spaces should be carefully examined as well. Signs such as loss of dorsal hair and redness may indicate significant ischemic changes. Palpation of the foot for warmth should be conducted as well. Peripheral pulsations such as dorsalis pedis and femoral artery should be palpated as well as, auscultated. Bruits are not uncommon in the femoral artery and early detection and management can prevent catastrophic complications. ${ }^{10}$

Monofilaments and bio-thesiometry should be used to test for peripheral neuropathy. If unavailable, physicians may test for light touch by cotton wool, pinprick, and vibration sense using a $128 \mathrm{~Hz}$ tuning fork. The aim is to detect whether the patient has developed peripheral neuropathy, which makes him more susceptible to DFU. ${ }^{11}$

Doppler studies often used to confirm the flow of the arterial pulses and gives impression of vascular patency and supply. When used with a sphygmomanometer, the ankle-brachial index can be measured. In normal subjects, the ankle systolic pressure is higher than the brachial systolic pressure. The normal ABI >1, a score $<0.9$ may indicate significant ischemia. Absent of pulses, with $\mathrm{ABI}<0.9$, confirm ischemia. While, a score $>1$ rules out ischemia. ${ }^{10}$

\section{Education}

Primary prevention is crucial in the prevention of DFU. In fact, up to $50 \%$ of DFU cases can be prevented by education. It is considered the cornerstone to prevent DFU. ${ }^{12}$ The goal of foot care education for diabetic patients are to prevent foot ulcers and amputation. A wide range of interventions exist to educate the patients toward better foot care to prevent DFU. It varies from demonstration to hands-on teaching. It is highly recommended that diabetic patients with DFU be educated about risk factors and the importance of foot care, including the self-inspection, monitoring foot temperature, daily foot hygiene, use of proper footwear, and blood sugar control. Studies showed that it is better to combine education with other care strategies. It can effectively reduce the incidence and morbidity of lower limb complications caused by DFU. ${ }^{13}$

\section{Blood sugar control}

In patients with DM, glucose control is one of the most important factors to prevent morbidities and mortalities. Inadequate control of blood glucose is the primary cause of DFU. ${ }^{14}$

One of the tests used to measure blood sugar is $\mathrm{HbA}_{1} \mathrm{C}$ level. This test indicates the average blood glucose concentration over about three months. The higher the $\mathrm{HbA}_{1} \mathrm{C}$ level, the more glycosylation of hemoglobin in red blood cells will occur. Studies have shown that blood glucose levels $>11.1 \mathrm{mmol} / \mathrm{L}$ (equivalent to $>310 \mathrm{mg} / \mathrm{mL}$ or an $\mathrm{HbA}_{1} \mathrm{C}$ level of $>12$ ) is associated with lower immunity because it decreases neutrophil function, including leukocyte chemotaxis. It also has a dose dependent effect; a higher blood glucose concentration has been associated with more inflammatory responses suppression and decreasing host response to an infection. ${ }^{14}$ Pomposelli et al, has showed that blood glucose level $>220 \mathrm{mg} / \mathrm{dL}$ on the first postoperative day is sensitive $(87.5 \%)$ predictor of postoperative infection. ${ }^{15}$ Moreover, the authors found that patients with blood glucose values $>220 \mathrm{mg} / \mathrm{dL}$ had infection rates that were 2.7 times higher than for patients with lower blood glucose values $(31.3 \%$ vs. $11.5 \%$, respectively). On the other hand, a $1 \%$ mean reduction in $\mathrm{HbA}_{1} \mathrm{C}$ was associated with a $25 \%$ reduction in micro vascular complications, including neuropathy. Poor glucose control also accelerates the process of PAD. In fact, every $1 \%$ increase in $\mathrm{HbA}_{1} \mathrm{C}$, there is an increase of $25 \%-28 \%$ in the relative risk of PAD, which is a primary cause of DFU. However, to date, no RCT has been performed to determine whether improved glucose control has benefits after a foot ulcer has developed. ${ }^{16}$

\section{Debridement}

Debridement is the surgical removal of the dead tissue, foreign, and infected materials from a wound. It is first and the most important intervention in the management of wound. It decreases the counts of bacteria, in addition it stimulates the production of local growth factors and reduces pressure. ${ }^{17}$

Different types of debridement are used including surgical, enzymatic, autolytic, mechanical, and biological. Surgical debridement has been considered the most effective in management of DFU. It involves cutting away dead and infected tissues followed by daily application of saline moistened cotton gauze. The main aim of surgical debridement is to change the pathology of a chronic ulcer to an acute one. Surgical debridement should be repeated on regular basis especially when new necrotic tissues occurs. Regular debridement has been shown to be associated with faster healing especially if it is conducted on every week. Successful debridement depends on several factors including characteristics, preferences, and practitioner level of expertise. When surgical or sharp debridement is not indicated, physicians 
should perform other types of debridement such as biological ones. ${ }^{18}$

Biological debridement includes maggot therapy (MDT) or larval therapy. It is used by applying a sterile and live forms of the Lucilia sericata on the wound to debride, disinfect, and heal the wound. The mechanism behind the larvae therapy is the secretion of a powerful autolytic enzyme that liquefies necrotic tissues, stimulates the healing processes, and destroys bacterial biofilms. It is indicated for open wounds and ulcers that contain gangrenous or necrotic tissues with or without infection. ${ }^{19}$

A few RCTs showed this method is effective in management of DFU. In addition to retrospective; and prospective studies have concluded that MDT is clinically effective treatment for DFU. The studies also showed significant decrease in wound odor and bacterial counts, such as methicillin-resistant Staphylococcus aureus. This has prevented hospital admission and decreased the number of outpatient visits in patients with DFU. $^{20}$

Although the advantages of biological debridement are evidence-based, adequate surgical debridement must always precede the application of topical wound healing agents, dressings, or wound closure procedures.

\section{Offloading}

Offloading also known as pressure modulation, is the most important method in the management of neuropathic ulcers in patients with DM. Studies have concluded the efficacy of this method in healing of DFU. Many methods exist, but only few studies were conducted to evaluate the rate of DFU healing. When choosing the proper method, the physicians have to consider many factors including the physical characteristics and the patient's compliance. Total contact casts (TCC) is the most effective offloading method for the management of the neuropathic DFU. TCC is shaped carefully to the foot with a heel for walking. ${ }^{21}$

The cast is designed to decrease the pressure on the ulcer and distribute it over the entire surface of the foot; thus, offer protection to the ulcer. Mueller et al conducted an RCT that concluded plantar ulcers heals at a faster rate when compared with the standard treatment. ${ }^{22}$ Histologic studies of the ulcers in patients treated with TCC has shown healing signs such as angiogenesis with the formation of granulation tissue which indicates better healing than patients treated with debridement alone. This can be probably attributed due to pressure redistribution and offloading. It also offers compliance because the patient cannot remove the cast thus reducing mobility. However, other studies reported side effects. Fife et al has shown that physicians rarely TCC in wound care in the united states. ${ }^{23}$ Only $16 \%$ of patients with DFU utilized TCC. This can be attributed to many disadvantages such as the need for expertise in its application. In adequate numbers of physicians or technicians might be a factor. Improper application may cause skin damage, pain, and irritation. Some patients reported the formation of new ulcers. In addition, the process of casting is time consuming, expensive, and it limits daily activities such as bathing. Moreover, TCC prevents daily visual assessment of the ulcer, which may be catastrophic in case of infections.

Other types of offloading methods exist such as removable cast walker (RCW) and instant TCC (iTCC). RCW works like a cast device that can be easily removed to let the patient self-inspect the ulcer and apply topical therapies. It allows the patients to bath and sleep comfortably. Adding to the benefits, they can be used in infected ulcers. To compare the effectiveness of TCC, RCW and half-shoe; a study was conducted to measure the healing time in each method. The healing rates were 33.5, 50.4, and 61.1 days, respectively. Significant number of patients with DFU were healed after 12 weeks of wearing TCC in comparison with the two other methods. $^{24}$

iTCC involves wrapping a RCW with a layer of cohesive bandage, elastoplast or casting tape, is another offloading method that was found to be more effective than TCC and RCW. This method prevents the patient from moving the foot, while allowing for ease of application and assessment of the ulcer when needed. ${ }^{25}$

In an RCT that was conducted to compare TCC to iTCC, it was found that there was no difference in effectiveness of iTCC and TCC in management of plantar neuropathic ulcer. In fact, iTCC was found to be cheaper and easier to apply by the patients. The study also reported fewer side effects. This method could revolutionize the management of plantar neuropathic ulcers. Other studies suggested that iTCC has the potential to replace TCC as the gold standard in the management of non-ischemic, neuropathic, diabetic plantar ulcer. ${ }^{25}$

Patients are advised to not return to regular shoes until the ulcer has healed completely. Moreover, it is highly recommended that any shoe that contributed to the formation of ulcer should not be used again. ${ }^{26}$

\section{Advanced dressing}

In the last decade, there was a breakthrough in the management of DFU especially with the demonstration of novel dressings. Dressing offers a moisturized balanced protection with protease sequestration and growth factor stimulation. Adding to these benefits, the antimicrobial, oxygen permeability and autolytic debridement helps produce granulation tissues and stimulates re-epithelialization. Furthermore, it has a longer time of action, higher efficacy and better release of medication therapies. ${ }^{27}$ However, each diabetic patient with DFU requires specific type of dressing. To choose the proper dressing, the physician has to determine 
several factors including the wound site, scar, depth, exudative vs. non-exudative, wound margins, presence of infection, and the dressing's conformability. In general, wound dressing are classified as passive, interactive, or active. While passive dressings are mainly used to protect the acute wounds as they absorb reasonable amounts of exudates and offer protection, the active and interactive dressings can modify the physiologic nature of the wound by promoting cellular and growth factors secretion. ${ }^{28}$

Dressings are also used for chronic ulcers as they easily adapt to wounds and preserve their moisture which helps in the healing process. Dressings used for DFU are: films, hydrogels, hydrocolloids, alginates, foams, and silverimpregnated. $^{27}$

All dressings are used in clinical practice, even there have been reported controversy about their effectiveness. Physicians are advised to choose according to several factors and based on each DFU. It was found that hydrogels have been the most popular choice of dressing for all DFU types. There are some studies that showed significant outcome in the management of DFU, but these studies were supported by incorporation of these products. However, these findings do not represent a practical option since the application of these compounds is expensive and difficult to regulate. Reports on benefits of hydrogels include longer wear times, greater absorbency, less painful, and less traumatic. In addition, they are cost effective in some patients because of the lowered rate of dressing changes and not requiring much nursing time. ${ }^{29}$

\section{Surgery}

For the past 2 decades, surgery has played a major role in the management of DFU as well as it's prevention. While surgery is not risk free, the selective correction of foot ulcers can better improve outcomes.

Generally, surgical procedures for DFU are categorized as non-vascular, vascular, and in some cases amputation. Nonvascular surgery is further categorized as elective, prophylactic, curative and emergent that aims to correct deformities. Long-term outcomes for diabetic foot surgery were evaluated in a few RCTs. Mueller et al conducted a study, in which patients were randomly assigned into two; achilles tendon-lengthening (ATL) group, received treatment of ATL and TCC, and a group who received TCC only. ${ }^{30}$ The results revealed that all ulcers healed in the ATL group and the risk for ulcer recurrence was $75 \%$ and $52 \%$ less at seven months and two years, respectively. ${ }^{30}$

Vascular foot surgery has shown to be effective in the management of ischemic foot ulcers. There is different approach to vascular surgery such as bypass grafts from femoral to pedal arteries and peripheral angioplasty, which improves blood flow in the foot. Some studies have recommended vascular foot surgery in ischemic foot, however, no RCT has been shown to reduce DFU. ${ }^{31}$

The primary goal of DFU management focuses on preserving the limb i.e. limb salvage, however in some cases amputation is necessary. In fact, it may offer a better outcome. Many factors may play role in making decision whether amputation should be performed, or other procedure can take place, such as patient's lifestyle, physical and psychological comorbidities. ${ }^{32}$

Generally, amputation is offered as the last resort after all other salvage techniques have been discussed, and the patient must agree. Some of the indications for an amputation include the removal of infected or gangrenous tissues, control of infection, and creation of a functional foot or stump that can accommodate footwear or prosthesis. $^{33}$

\section{CONCLUSION}

DFU is a common morbidity in patients with DM. It frequently leads to lower limb amputation unless a prompt, rational, multidisciplinary approach to therapy is taken. Proper management can ensure successful and fast healing of DFU. It includes patient education, blood glucose control, wound debridement, advanced dressing, offloading, and surgery. Management should be used frequently to decrease the morbidity and risk of different complications the result from foot ulcers.

\section{Funding: No funding sources \\ Conflict of interest: None declared \\ Ethical approval: Not required}

\section{REFERENCES}

1. Whiting DR, Guariguata L, Weil C, Shaw J. IDF diabetes atlas: global estimates of the prevalence of diabetes for 2011 and 2030. Diabetes Res Clin Pract. 2011;94:311-21.

2. Nather A et al. Epidemiology of diabetic foot problems and predictive factors for limb loss. J Diabetes Complications. 2008;22:77-82.

3. Snyder RJ, Hanft JR. Diabetic foot ulcers-effects on QOL, costs, and mortality and the role of standard wound care and advanced-care therapies. Ostomy Wound Manage. 2009;55:28-38.

4. Bowering CK. Diabetic foot ulcers. Pathophysiology, assessment, and therapy. Can Fam Physician. 2001;47:1007-16.

5. Kelkar P. Diabetic neuropathy. Semin Neurol. 2005;25:168-73.

6. Thiruvoipati T, Kielhorn CE, Armstrong EJ. Peripheral artery disease in patients with diabetes: Epidemiology, mechanisms, and outcomes. World J Diabetes. 2015;6:961-9.

7. Hobizal KB, Wukich DK. Diabetic foot infections:current concept review. Diabet Foot Ankle. 2012;3. 
8. Malhotra R, Chan CS, Nather A. Osteomyelitis in the diabetic foot. Diabet Foot Ankle. 2014;5.

9. Yuh WT, Corson JD, Baraniewski HM, Rezai K, Shamma AR, Kathol MH, et al. Osteomyelitis of the foot in diabetic patients:evaluation with plain film, 99mTc-MDP bone scintigraphy, and MR imaging. Am J Roentgenol. 1989;152:795-800.

10. Kravitz SR, McGuire J, Shanahan SD. Physical assessment of the diabetic foot. Adv Skin Wound Care. 2003;16:68-75.

11. Boulton AJ, Armstrong DG, Albert SF, Frykberg RG, Hellman R, Kirkman MS, et al. Comprehensive foot examination and risk assessment: a report of the task force of the foot care interest group of the American Diabetes Association, with endorsement by the American Association of Clinical Endocrinologists. Diabetes Care. 2008;31:1679-85.

12. Iraj B, Khorvash F, Ebneshahidi A, Askari G. Prevention of diabetic foot ulcer. Int J Prev Med. 2013;4:373-6.

13. Dorresteijn JA, Kriegsman DM, Assendelft WJ, Valk GD. Patient education for preventing diabetic foot ulceration. Cochrane Database Syst Rev. 2012; doi:10.1002/14651858.CD001488.pub4.

14. McMurry JF Jr. Wound healing with diabetes mellitus. Better glucose control for better wound healing in diabetes. Surg Clin North Am. 1984;64:769-78.

15. Pomposelli JJ, Baxter JK 3rd, Babineau TJ, Pomfret EA, Driscoll DF, Forse RA, et al. Early postoperative glucose control predicts nosocomial infection rate in diabetic patients. JPEN J Parenter Enteral Nutr. 1998;22:77-81.

16. UK Prospective Diabetes Study (UKPDS) Group. Effect of intensive blood-glucose control with metformin on complications in overweight patients with type 2 diabetes (UKPDS 34). Lancet. 1998;352:854-65.

17. DiPreta JA. Outpatient assessment and management of the diabetic foot. Med Clin North Am. 2014;98:353-73.

18. Lebrun E, Tomic-Canic M, Kirsner RS. The role of surgical debridement in healing of diabetic foot ulcers. Wound Repair Regen. 2010;18:433-8.

19. Mumcuoglu KY. Clinical applications for maggots in wound care. Am J Clin Dermatol. 2001;2:219-27.

20. Sherman RA. Maggot therapy for treating diabetic foot ulcers unresponsive to conventional therapy. Diabetes Care. 2003;26:446-51.

21. Boulton AJ. Pressure and the diabetic foot: clinical science and offloading techniques. Am J Surg. 2004; 187:17-24.

22. Mueller MJ, Diamond JE, Sinacore DR, Delitto A, Blair VP 3rd, Drury DA, et al. Total contact casting in treatment of diabetic plantar ulcers. Controlled clinical trial. Diabetes Care. 1989;12:384-8.

23. Fife CE, Carter MJ, Walker D, Thomson B, Eckert KA. Diabetic foot ulcer off-loading: The gap between evidence and practice. Data from the US Wound Registry. Adv Skin Wound Care. 2014;27:310-6.

24. Armstrong DG, Nguyen HC, Lavery LA, van Schie $\mathrm{CH}$, Boulton AJ, Harkless LB. Off-loading the diabetic foot wound: a randomized clinical trial. Diabetes Care. 2001;24:1019-22.

25. Katz IA, Harlan A, Miranda-Palma B, PrietoSanchez L, Armstrong DG, Bowker JH, et al. A randomized trial of two irremovable off-loading devices in the management of plantar neuropathic diabetic foot ulcers. Diabetes Care. 2005;28:555-9.

26. Singh N, Armstrong DG, Lipsky BA. Preventing foot ulcers in patients with diabetes. JAMA. 2005;293:217-28.

27. Moura LI, Dias AM, Carvalho E, de Sousa HC. Recent advances on the development of wound dressings for diabetic foot ulcer treatment-a review. Acta Biomater. 2013;9:7093-114.

28. Hansson C. Interactive wound dressings. A practical guide to their use in older patients. Drugs Aging. 1997;11:271-84.

29. Dumville JC, O'Meara S, Deshpande S, Speak K. Alginate dressings for healing diabetic foot ulcers. Cochrane Database Syst Rev. 2012; doi:10.1002/14651858.CD009110.pub2.

30. Mueller MJ, Sinacore DR, Hastings MK, Strube MJ, Johnson JE. Effect of Achilles tendon lengthening on neuropathic plantar ulcers. A randomized clinical trial. J Bone Joint Surg Am. 2003;85:1436-45.

31. Sumpio BE, Lee T, Blume PA. Vascular evaluation and arterial reconstruction of the diabetic foot. Clin Podiatr Med Surg. 2003;20:689-708.

32. Lepantalo M, Apelqvist J, Setacci C, Ricco JB, de Donato G, Becker F, et al. Chapter V:Diabetic foot. Eur J Vasc Endovasc Surg. 2011;42:60-74.

33. Abou-Zamzam AM Jr., Gomez NR, Molkara A, Banta JE, Teruya TH, Killeen JD, et al. A prospective analysis of critical limb ischemia:factors leading to major primary amputation versus revascularization. Ann Vasc Surg. 2007;21:458-63.

Cite this article as: Alsanawi $\mathrm{Y}$, Alismail $\mathrm{H}$, AlabdRabalnabi M, Alturki H, Alsuhaibani A, Mahbub M, et al. Pathogenesis and management of diabetic foot ulcers. Int J Community Med Public Health 2018;5:4953-8. 